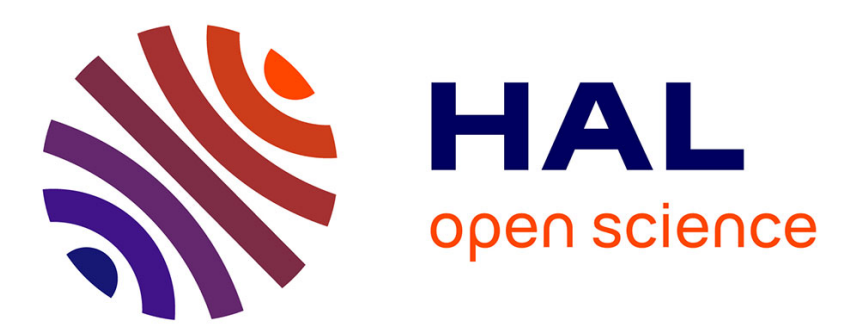

\title{
Bases de la simulation en santé et exemple en santé au travail
}

\author{
A. Bellahouel, A. Descatha
}

\section{To cite this version:}

A. Bellahouel, A. Descatha. Bases de la simulation en santé et exemple en santé au travail. Archives des Maladies Professionnelles et de L'Environnement, 2019, 80 (2), pp.133-138. 10.1016/j.admp.2018.11.005 . hal-02153436

\section{HAL Id: hal-02153436 https://hal-univ-rennes1.archives-ouvertes.fr/hal-02153436}

Submitted on 22 Oct 2021

HAL is a multi-disciplinary open access archive for the deposit and dissemination of scientific research documents, whether they are published or not. The documents may come from teaching and research institutions in France or abroad, or from public or private research centers.
L'archive ouverte pluridisciplinaire HAL, est destinée au dépôt et à la diffusion de documents scientifiques de niveau recherche, publiés ou non, émanant des établissements d'enseignement et de recherche français ou étrangers, des laboratoires publics ou privés.

\section{다)(1) $(5$}

Distributed under a Creative Commons Attribution - NonCommerciall 4.0 International 
Archives des Maladies Professionnelles et de l'Environnement

MISE AU POINT- Développement professionnel continu

\title{
Bases de la simulation en santé et exemple en santé au travail
}

\section{Fundamentals of Healthcare Simulation training and example of an application}

\section{in occupational health}

\section{A. BELLAHOUEL ${ }^{a}$, A.DESCATHA ${ }^{b}$}

a Service de santé au travail, Hôpitaux Universitaires Paris Ouest, Hôpital Européen

Georges Pompidou AP-HP 75908 Paris Cedex 15, France

b Unité hospitalo-universitaire de santé professionnelle, Samu92, Inserm UMS011 U1168

U1085, CHU Poincaré, AP-HP, 92380 Garches, France

\author{
${ }^{*}$ Auteur correspondant \\ Adresse e-mail : amel.bellahouel@aphp.fr (A.BELLAHOUEL)
}

Mots clés: pédagogie ; santé au travail ; Simulation en santé ; Soins d'urgence en milieu du travail

Keywords : education; occupational health; healthcare simulation; emergency care in the workplace 


\section{INTRODUCTION}

La réforme du 3ème cycle des études médicales entrée en vigueur en 2017, encourage l'utilisation de méthodes pédagogiques innovantes en privilégiant entre autre, les méthodes de simulation en santé (1). Le déploiement de cet outil pédagogique innovant pour la formation des internes, s'est fait à travers des cas cliniques de simulation humaine, synthétique sur mannequin basse fidélité et numérique par la création d'un jeu sérieux (serious game) adapté à la spécialité. L'émergence et le déploiement de ces méthodes intéressent de plus en plus le monde de la santé dans toutes les disciplines.

L'objectif est de présenter une mise au point sur les principes généraux de la simulation en santé et détailler un exemple en santé au travail, celui de l'urgence. En effet, dans ce contexte de réforme, une modification du programme d'enseignement du module 6 « soins d'urgence » du DES de médecine du travail en lle de France a été décidé. Partis de nos expériences de médecins urgentistes et de formateur à l'enseignement de la médecine sur simulateur pour essayer de répondre aux objectifs pédagogiques de la nouvelle maquette.

\section{La simulation en santé}

\section{* Définition et principes}

Selon I'HAS (2) : Le terme Simulation en santé correspond " à l'utilisation d'un matériel (comme un mannequin ou un simulateur procédural), de la réalité virtuelle ou d'un patient standardisé pour reproduire des situations ou des environnements de soin, dans le but d'enseigner des procédures diagnostiques et thérapeutiques et de répéter des processus, des concepts médicaux ou des prises de décision par un professionnel de santé ou une équipe de professionnels ».

Comme les simulateurs de vols pour les pilotes, la formation par la simulation en médecine et santé est un outil innovateur d'apprentissage par la pratique. II permet par une mise en situation à l'apprenant, d'effectuer des actes médicaux ou des soins semblables à la réalité.

Le déroulement d'une séance de simulation (2) doit obéir au schéma suivant :

- Briefing, indispensable afin d'expliquer le déroulement de la séance, créer un climat de confiance favorisant l'apprentissage, propulser les apprenants dans le contexte de la situation simulée

- Pratique Simulée 
- Débriefing, temps majeur d'apprentissage et de réflexion comportant une phase de réaction, une phase d'analyse et une phase de conclusion

- Conclusion de séance

Les critères qualifiant une méthode pédagogique par la simulation (3) sont les suivants :

- Un objectif pédagogique

- Un scénario bâti et rédigé visant cet objectif

- Un briefing de présentation du contexte et de l'équipement

- Un environnement réaliste (validation de la capacité de l'environnement à atteindre l'objectif pédagogique : équipement, réalisme, matériel, mannequin)

- Un débriefing structuré et encadré

- Un document de fin de séance ou fiche d'aide à la progression

\section{* Objectifs pédagogiques}

Les objectifs pédagogiques de cette formation, qui s'adaptent aussi bien aux étudiants qu'aux professionnels, se regroupent sous 2 catégories :

- l'apprentissage et acquisition des compétences médicales et des gestes techniques

- l'analyse des compétences non techniques ou CRM : gestion de crise, communication, travail d'équipe, appel à l'aide, leadership...

\section{* Différents techniques de simulation en santé}

On distingue différentes techniques de simulation en santé $(4,5,6)$; (Schéma 1) :

- Humaine : patient standardisé, jeu de rôles

- Synthétique : Simulateurs patients, Simulateurs procéduraux (mannequins haute et basse fidélité)

- Electronique : Réalité virtuelle, Environnement 3D et jeux sérieux

\section{La simulation humaine}


La simulation humaine suit le principe d'un jeu de rôle, le but étant de mettre l'apprenant en face d'un patient standardisé afin de suivre la relation soignant-soigné.

Un patient standardisé est une personne qui joue le rôle d'un patient, selon une situation scénarisée (comportements et situations cliniques définies à l'avance). II peut donc retranscrire les réactions émotionnelles et la personnalité d'un patient, ce que ne permet pas la simulation synthétique, et donc de pouvoir étudier la relation soignant-soigné et de l'améliorer.

\section{La simulation synthétique}

\section{Mannequin de simulation haute-fidélité}

Les mannequins « haute fidélité » permettent de s'approcher au plus près de la réalité.

Ils reproduisent des situations de détresse (parler, transpirer, saigner, devenir cyanosé, etc.) en interagissant en temps réel avec les apprenants. Ces derniers peuvent prendre des décisions

thérapeutiques et effectuer des gestes techniques (perfuser, ventiler, intuber, masser, drainer, sonder, accoucher) adaptés.

II existe différents mannequins hautes fidélités : mannequins adultes, mannequins pédiatriques, mannequins nouveau-nés, mannequins d'accouchement, mannequins enfants...

\section{Mannequin basse-fidélité}

Ces mannequins sont adaptés à l'apprentissage de gestes techniques simples comme le massage cardiaque, la perfusion, la ponction lombaire, la suture, la ventilation, l'intubation...

\section{La simulation électronique}

La simulation électronique se caractérise par l'utilisation d'un support informatique ou multimédia pour mettre en situation un cas prédéfini.

\section{$\underline{\text { Serious Game }}$}

Le jeu sérieux est une forme de simulation électronique dont la création requiert la définition préalable d'objectifs pédagogiques que le jeu mettra en lumière. II s'agit d'un jeu vidéo qui met l'apprenant en immersion virtuelle, et lui permet d'acquérir des compétences et des connaissances. 


\section{Enseignement de la gestion des urgences en milieu de travail}

\section{* $\quad$ Rôle du médecin du travail}

La gestion des urgences est de la responsabilité du médecin du travail avec son équipe (7) qu'il soit présent ou non, même s'il peut s'appuyer sur les systèmes d'urgence.

La survenance d'un accident du travail, d'une détresse médicale ou d'un état pathologique dans l'entreprise nécessite la mise en place de moyens efficaces destinés à prendre en charge le plus rapidement possible la victime. L'organisation des secours passe par la mise en œuvre de moyens humains, la formalisation de la conduite à tenir en cas d'urgence ou d'accident, un dispositif d'alerte efficace et la mise à disposition de moyens de secours adaptés dans l'entreprise.

Une gestion adéquate de l'urgence en milieu de travail optimise le pronostic et le retour à l'emploi d'une victime, permettant ainsi au médecin du travail d'exercer autant son rôle propre dans la prévention primaire en anticipant cette organisation, que secondaire et tertiaire.

Afin d'assurer pleinement son rôle dans une gestion appropriée de l'urgence, le médecin du travail doit acquérir lors de sa formation initiale les connaissances théoriques nécessaires et une approche pédagogique pratique basée sur l'entraînement afin de développer des réflexes, qu'il doit maintenir tout au long de son exercice professionnel.

\section{Exemple de déploiement d'un programme de simulation dans la gestion de l'urgence en santé au travail}

\subsection{Généralités}

La filière du Diplôme d'Etudes Spécialisées (DES) en Médecine du Travail comporte un enseignement théorique de 250 heures réparties en 7 modules :

Concernant le module 6 « soins d'urgence », l'objectif de la réforme de la maquette du $3^{\text {ème }}$ cycle est de maîtriser le principe d'organisation des secours d'urgence en fonction des entreprises et du contexte sanitaire extérieur. Au sein de la promotion d'étudiants pour l'année 2018, concernés par l'enseignement du module 6 " soins d'urgence », cohabitaient des internes de phase socle, des internes anciens régimes, des collaborateurs médecins et des PAE.

Afin d'adapter au mieux cet enseignement aux besoins spécifiques de chaque catégorie d'étudiants tout en répondant aux objectifs de la nouvelle réforme, il a été nécessaire de 
mettre en place un nouveau programme sous forme d'un parcours pédagogique permettant d'intégrer l'ensemble des étudiants juniors et séniors.

L'objectif est de décrire la mise en place ce nouveau programme d'enseignement en tenant compte des objectifs de la réforme des études médicales du $3^{\text {ème }}$ cycle puis d'évaluer la qualité de cet enseignement de deux façons, par :

- Une enquête de satisfaction générale auprès des étudiants avec analyse de l'intérêt perçu pour ce type d'outil pédagogique innovant dans la formation de futur médecin du travail

- Un questionnaire comportant 12 questions à choix multiple complété avant et après enseignement afin de juger de l'amélioration de leurs connaissances et donc de l'efficacité de ce nouveau format

\subsection{Nouveau programme}

Le nouveau programme d'enseignement du module « soins d'urgence » en lle de France pour l'année 2018 s'est déroulé en deux journées: une de préparation et de restitution du travail de groupe et une de mise en pratique à travers deux cas cliniques de simulation et un jeu sérieux. Son évaluation s'est faite par les deux enquêtes sus cités (QCM d'évaluation avant / après enseignement et questionnaire de satisfaction).

Les objectifs étaient d'/de :

- Acquérir des notions physiopathologiques précises autour d'une douzaine de thèmes d'urgence en apportant deux références bibliographiques de bonne qualité par thème en français et en anglais pour les internes de phase socle

- Comprendre les bases de réalisation de protocoles d'urgence en présence ou non de professionnel de santé pour l'ensemble de la promotion.

- Mettre en pratique ces connaissances à travers :

- Deux scénarios de cas cliniques de simulation selon des objectifs pédagogiques prédéfinis, sur le principe du volontariat selon le schéma du briefing - pratique simulée - débriefing et conclusion.

Le premier scénario, traitait d'un cas de douleur thoracique sur le lieu de travail compliquée d'un arrêt cardiaque et le second, d'un cas d'accident vasculaire cérébral chez un salarié alcoolisé agité.

Le but était d'amener les étudiants à comprendre et appliquer les bases de la 
méthode de réalisation de protocoles en entreprise, de développer le travail en équipe et la communication avec les partenaires de l'urgence internes à l'entreprise et les secours externes.

- La simulation d'un jeu sérieux (serious game) portant sur un cas clinique d'asthme professionnel sur syndrome de Brooks chez un salarié agent de nettoyage, en présence du médecin du travail.

Le but était d'amener les étudiants à mettre en lumière par le jeu les objectifs pédagogiques prédéfinis. Le jeu noté sur un total de 100 points comporte :

1. Cadre général portant sur la situation clinique

2. Phase 0 : décision d'orientation médicale

3. Phase I : état de base axé sur l'interrogatoire, l'orientation diagnostique

4. Phase II: aggravation avec des signes cliniques et paracliniques de défaillance respiratoire à identifier pour décider du moment de l'appel au médecin régulateur du SAMU

5. Dialogue et premières thérapeutiques clefs à initier en attendant l'arrivée des secours

Chaque phase, inclut des éléments « éliminatoires " invitant l'étudiant à reprendre le jeu. Des feedbacks sont prévus pour lui rappeler les objectifs pédagogiques. Son propre score et ceux obtenus par d'autres joueurs sont enregistrés l'encourageant ainsi à progresser. Un débriefing final comportant des référentiels sur la pathologie et une synthèse du cas clinique sont accessibles en fin de jeu.

En amont du séminaire, des supports de références sur les thématiques qui seront traitées pendant l'enseignement, ont été mis à disposition des étudiants afin de faciliter le travail de groupe demandé, d'acquérir de nouvelles connaissances et de renforcer celles de base. Ces supports comportaient trois vidéos traitant de la gestion des urgences en milieu de travail, accessibles sur la plateforme numérique de santé et différents articles portant sur la réglementation, la lecture de l'ECG et les bases de réalisation de protocoles.

L'intérêt et le principe de l'enseignement par méthode de simulation sont expliqués au préalable aux étudiants, y compris pour le jeu sérieux.

\section{$\underline{3.3 \text { Evaluation du nouveau programme d'enseignement }}$}


Deux outils ont permis d'évaluer la qualité de la nouvelle maquette d'enseignement.

\section{- Questionnaire d'évaluation}

II s'agit d'un questionnaire comportant 12 questions à choix multiple complété par les étudiants avant que l'enseignement ne soit dispensé, puis après qu'il soit terminé. L'analyse des réponses des étudiants avant l'enseignement permettra d'évaluer leur niveau de base puis la comparaison avec celles rendues après enseignement permettra de juger de l'amélioration de leurs connaissances et donc de l'efficacité de ce nouveau format d'enseignement pour la gestion de situations d'urgence en milieu de travail.

Une correction du questionnaire avec l'ensemble de la promotion juste avant la clôture de l'enseignement a permis de revenir une fois de plus sur les objectifs pédagogiques de l'enseignement et favoriser l'échange entre étudiants et formateurs afin d'éviter toute incompréhension ou confusion.

\section{- Questionnaire de satisfaction}

Une enquête de satisfaction a été soumise aux étudiants à l'issue de la formation.

Ses objectifs sont de :

- Connaître l'expérience des étudiants juniors et séniors durant leurs cursus concernant le domaine de la simulation

- Juger de l'intérêt global de la formation, de l'intérêt perçu des différents scénarios, du degré de réalisme de la situation clinique, du degré de stress ressenti durant le scénario, de l'éventualité de concevoir une fin de scénario par un décès et de l'intérêt perçu de la simulation pour la pratique

- Juger de l'intérêt perçu de cet outil pédagogique en vue de nouvelles séances de formation par la simulation synthétique, les jeux sérieux

- Juger de la place de la simulation dans la formation médicale et l'évaluation des pratiques

Le questionnaire de satisfaction comprend 5 parties avec une à plusieurs questions par partie et un commentaire libre. Certaines questions sont à réponses binaires (oui ou non), d'autres appellent à une réponse de 1 (pas intéressant) à 4 (très intéressant) permettent d'obtenir un score ou indice moyen de satisfaction

Ainsi, 71 questionnaires ont été analysés, un grand nombre d'étudiants (72) ont participé aux 
deux journées de séminaire, dont certains ayant déjà une première expérience de simulation en contexte médical.

Afin de juger de l'efficacité spécifique de cette nouvelle maquette, les résultats ont permis de calculer les moyennes avant et après l'enseignement et mesurer son impact sur les connaissances en soins d'urgence des étudiants. Ces moyennes ont été calculées pour l'ensemble des étudiants puis séparément pour le groupe d'étudiants avec expérience de simulation et comparées au groupe d'étudiants sans expérience.

Les moyennes sont représentées en figure 1 , avec une amélioration significative $(p<0.05)$.

Figure 1: Distribution globale des résultats avant et après enseignement

Le questionnaire de satisfaction a été distribué aux étudiants à la fin de l'enseignement. Pour chaque item, une note de 1 à 4 (Pas intéressant $=1$, Moyen $=2$, Intéressant $=3$, Très intéressant $=4$ ) devait être attribuée, afin d'établir pour chacun un indice moyen de satisfaction.

Les résultats de ce questionnaire de satisfaction sont excellents (Tableau 1). La figure 2 et 3 
Tableau 1 : Résultat du questionnaire de satisfaction.

\begin{tabular}{|l|l|}
\hline Questions & réponses \\
\hline Q1 - Intérêt des différents scénarios : & $3,7 / 4$ \\
Q3- Déficit neurologique / salarié alcoolisé et agité & $3,7 / 4$ \\
Q4- Asthme professionnel / syndrome de Brooks & $3,7 / 4$ \\
Q5- Améliorer la prise en charge des patients & $3,6 / 4$ \\
Q6- Se confronter à des évènements rares & $3,5 / 4$ \\
Q7- Apprendre à gérer une équipe & $3,5 / 4$ \\
Q8 Votre degré de stress durant le scénario & $3,4 / 4$ \\
\hline
\end{tabular}

Figure 2: Distribution des appréciations pour l'item « intérêt pour de nouvelles formations par simulation/ jeux sérieux 
Figure 3: Distribution des appréciations pour l'item « formation et évaluation

La forte participation des étudiants à l'évaluation du nouveau programme d'enseignement a permis d'avoir des moyennes représentatives afin de juger de la satisfaction moyenne et d'évaluer leur niveau. La significativité des résultats semble indiquer une amélioration des connaissances dans la gestion des urgences en milieu de travail grâce à ce format d'enseignement malgré le fait qu'il soit jugé court.

Par ailleurs cette évaluation a permis de mettre en avant, une différence de niveau entre étudiants ayant une expérience en simulation même unique et ceux sans expérience, confirmant le rôle que peut jouer ce type d'outil pédagogique dans la consolidation des connaissances ; malgré tout, cela nécessiterait une pratique plus régulière pour améliorer la rétention des acquis à long terme.

Enfin les indices de satisfaction très élevés selon le modèle d'évaluation de Kirkpatrick (10), encouragent à développer ce type d'enseignement.

II est important de noter que l'évaluation à chaud du ressenti des étudiants découvrant pour la première fois, ce type d'outil pédagogique innovant peut représenter une surestimation de leur satisfaction de façon globale.

L'échantillon d'étudiants ayant une expérience antérieure de simulation est faible pour être représentatif ; et pour certains l'expérience acquise remonte à un certain temps ce qui peut biaiser les résultats. 
La nécessité de formateurs qualifiés en simulation avec des compétences validées par l'obtention de diplômes universitaires spécifiques, le temps et l'investissement nécessaires pour mettre en place des enseignements par simulation peuvent représenter un frein au déploiement de ce type d'outil.

Ainsi, la satisfaction, quant au réalisme des scénarios et l'intérêt perçu de l'inclusion de la simulation dans leur formation, encouragent à développer ces outils pédagogiques. L'intégration du jeu sérieux dans le cursus de formation des étudiants de la spécialité via la plateforme numérique en complément des enseignements existants, permettra de répondre d'une part à un besoin de formation pour l'ensemble des étudiants sur le territoire national, notamment dans un contexte où le nombre d'enseignants est en diminution et d'autre part à l'enregistrement des traces d'apprentissage jusqu'à l'atteinte des objectifs pédagogiques prédéfinis tout en développant la démarche réflexive des apprenants voulue par la réforme.

\section{Perspectives}

La simulation en santé, représente un outil pédagogique d'amélioration des compétences techniques (11), non techniques (12) et peut s'appliquer à toutes les spécialités (13) ; elle trouve toute sa place dans le cursus de formation du futur médecin du travail autant en formation initiale que dans le cadre du développement professionnel continu (DPC).

Liens d'intérêts : Les auteurs sont payés par les institutions. Alexis Descatha est rédacteur en chef des Archives des maladies professionnelles et de l'environnement.

\section{REFERENCES}

1. Arrêté du 21 avril 2017 relatif aux connaissances, aux compétences et aux maquettes de formation des diplômes d'études spécialisées et fixant la liste de ces diplômes et des options et formations spécialisées transversales du troisième cycle des études de médecine.

2. Haute Autorité de Santé - Simulation en santé [Internet]. [cité 9 sept 2018]. Disponible sur: https://www.has-sante.fr/portail/jcms/c_930641/fr/simulation-en-sante

3. Boet S, Granry J-C, Savoldelli G. La simulation en santé: De la théorie à la pratique. Springer Science \& Business Media; 2013. 439 p.

4. classification des techniques de simulation chiniara - Recherche Google [Internet]. [cité 9 sept 2018]. Disponible sur: https://www.google.fr/search?ei=54OVW_yPAYm2adK8qJAP\&q=classification+des+techniques+de 
+simulation+chiniara\&oq=classification+des+techniques+de+simulation+chiniara\&gs_l=psy-ab.

5. Alinier G. A typology of educationally focused medical simulation tools. 2007 [cité 9 sept 2018]; Disponible sur: http://uhra.herts.ac.uk/handle/2299/2335

6. nhs simulation provision and use study london department of health - Recherche Google [Internet]. [cité 9 sept 2018]. Disponible sur: https://www.google.fr/search?ei=S2eVW-r5KoaacTOldAF\&q=nhs+simulation+provision+and+use+study+london+department+of+health\&oq=nhs+si mulation+provision+and+use+study+london+department+of+health\&gs_l=psyab.3...4293.26337.0.27385.46.39.7.0.0.0.459.3862.23j15j4-1.39.0..2..0...1.1.64.psyab..0.32.3426...0i22i30k1j33i21k1j33i160k1j33i10k1.0.pah1pxHhg5I

7. Chamoux A, Dang C, Soula MC, Dolveck F, Havette P, Coudurier F, et al. Place du médecin du travail dans l'organisation des urgences en milieu de travail.

/data/revues/17758785/v70i2/S1775878509000289/ [Internet]. 7 mai 2009 [cité 9 sept 2018];

Disponible sur: http://www.em-consulte.com/en/article/212319

8. Fanning RM, Gaba DM. The Role of Debriefing in Simulation-Based Learning: Simulation in Healthcare: The Journal of the Society for Simulation in Healthcare. 2007;2(2):115 25.

9. Renou J. Le débriefing en simulation médicale: techniques et outils pédagogiques. :59.

10. Le modèle de Kirkpatrick [Internet]. [cité 9 sept 2018]. Disponible sur: https://www.kirkpatrickpartners.com/Our-Philosophy/The-Kirkpatrick-Model

11. Mundell WC, Kennedy CC, Szostek JH, Cook DA. Simulation technology for resuscitation training: A systematic review and meta-analysis. Resuscitation. 1 sept 2013;84(9):1174 83.

12. Yee B, Naik VN, Joo HS, Savoldelli GL, Chung DY, Houston PL, et al. Nontechnical Skills in Anesthesia Crisis Management with Repeated Exposure to Simulation-based Education. Anesthes. 1 août 2005;103(2):241 8 .

13. Granry J-C. La simulation en santé : quels enjeux ? Revue des Maladies Respiratoires. déc 2015;32(10):966 


\section{Points essentiels}

- La simulation en santé représente un outil pédagogique innovant pour l'amélioration des compétences techniques, non techniques (CRM) et s'applique à toutes les spécialités.

- La gestion adéquate de l'urgence en milieu de travail optimise le pronostic et le retour à l'emploi d'une victime et renfonce le rôle du médecin du travail dans la prévention primaire ainsi que secondaire et tertiaire.

- La réforme du 3 ème cycle des études médicales appelle à renforcer le rôle du médecin quel que soit sa spécialité dans la gestion de l'urgence et encourage le recours à des méthodes pédagogiques innovantes telle que la simulation en santé.

- La simulation en santé trouve toute sa place dans le cursus de formation du futur médecin du travail autant en formation initiale que dans le cadre du développement professionnel continu (DPC). 
Figure 1: Distribution globale des résultats avant et après enseignement

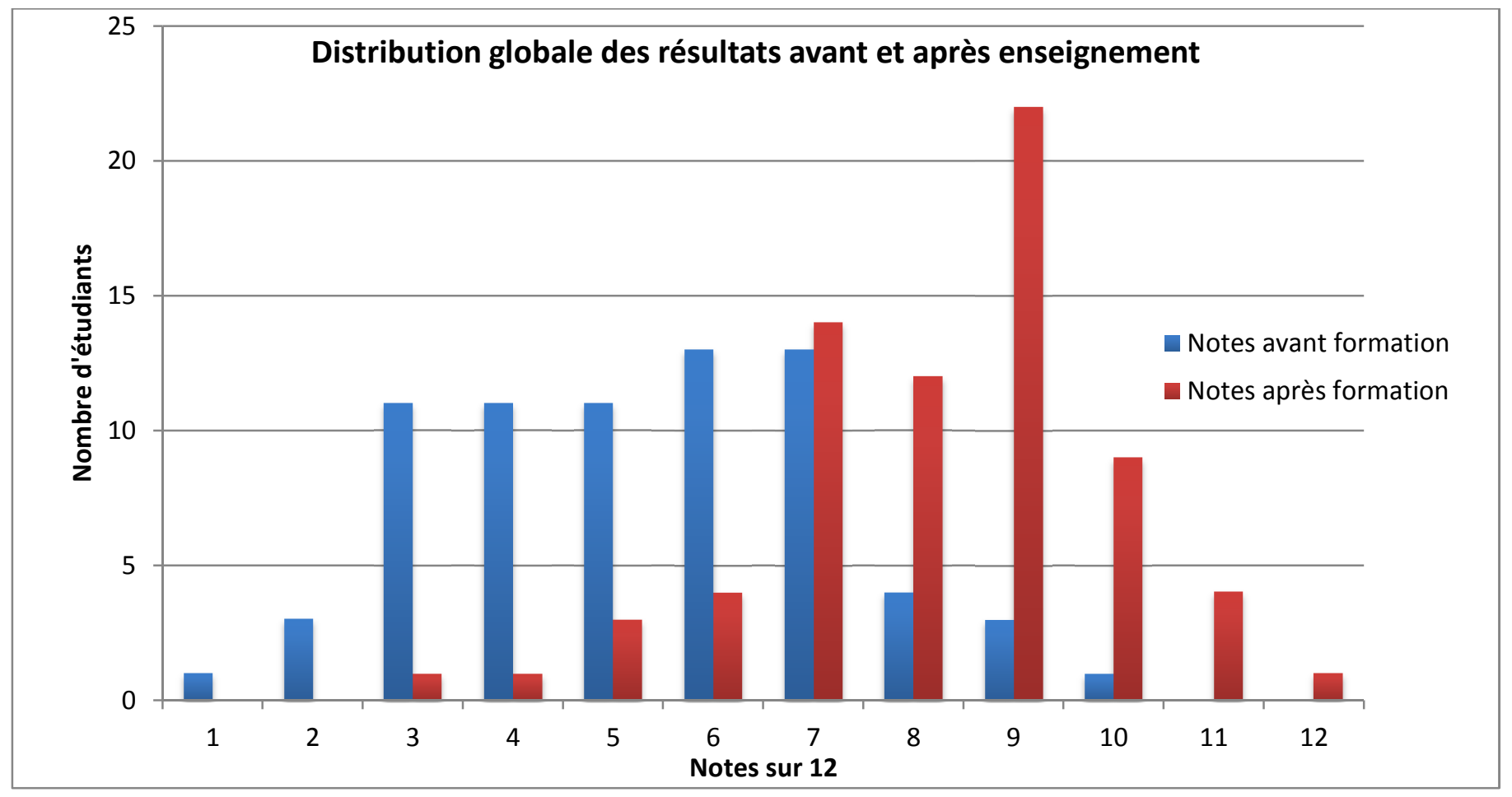

Figure 2: Distribution des appréciations pour l'item « Intérêt global de la formation » et les différents scénarios

Distribution des appréciations pour l'item « Intérêt global de la formation » et les différents scénarios

60

50

40

30

20

10

0

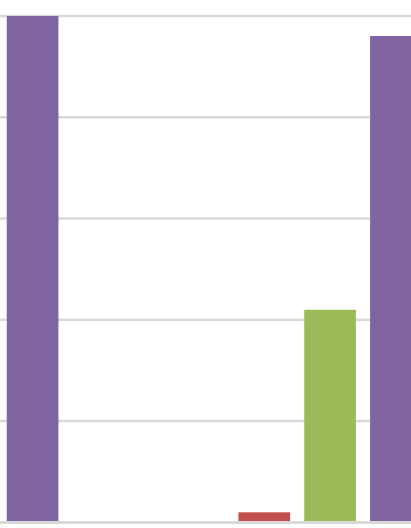

Q2

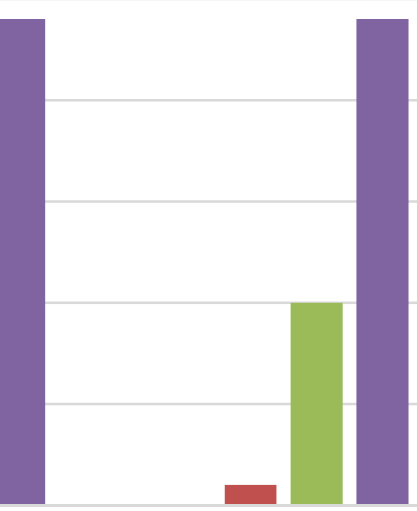

Q3

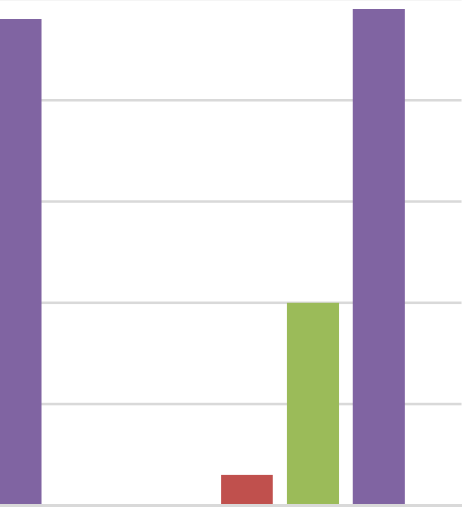

Q4

• Pas intéressant $\square$ Moyennement intéressant $\quad$ Intéressant $\square$ Très intéressant 
Figure 3 : Distribution des appréciations pour l'item « formation et évaluation »

Pensez-vous qu'une séance de simulation puisse faire partie de la formation médicale initiale ou continue?

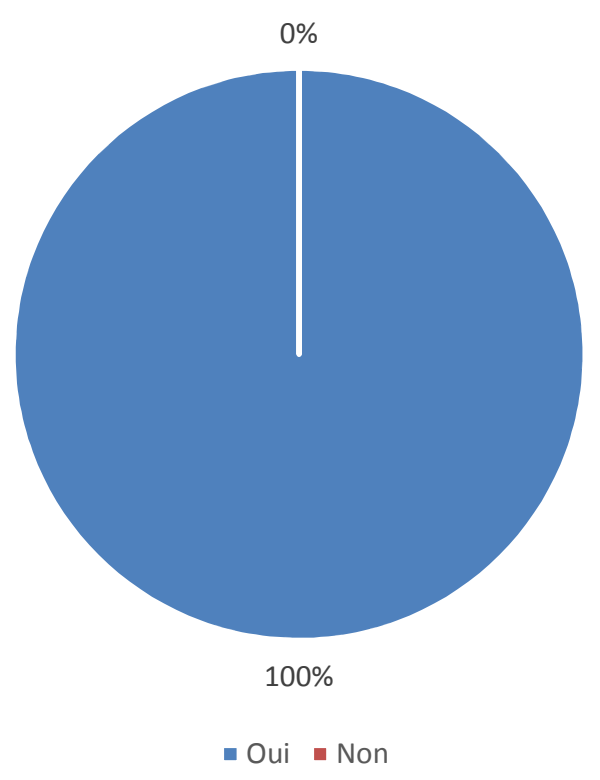


Schéma 1: Les différentes techniques de simulation en santé 